\title{
Bumetanide in Hypokalaemic Periodic Paralysis: a randomised, double-blind, placebo controlled phase II clinical trial with a crossover design
}

Renata S. Scalco, Iwona Skorupinska, Camille Blochet, Mariam Habib, Emma Matthews, Jasper Morrow, Michael G. Hanna, Doreen Fialho

MRC Centre for Neuromuscular Diseases, Institute of Neurology, UCL, London, United Kingdom

Email: r.scalco@ucl.ac.uk

Background: Hypokalamic periodic paralysis (hypoPP) is a muscle channelopathy characterised by episodes of focal or generalised attacks of weakness accompanied by low serum potassium. Treatment to abort acute attacks is limited to potassium supplement. Recent animal studies suggested that bumetanide may be useful in both preventing and aborting an acute attack of weakness. We therefore designed a clinical trial to explore possible efficacy and safety aspects of bumetanide in patients with genetically confirmed hypoPP.

Method: Randomised double blind placebo controlled phase II clinical trial with a crossover design carried out at the MRC Centre for Neuromuscular Diseases, London, aiming to recruit a total of 12 participants. A focal attack of HypoPP is provoked by exercise in a hand muscle (using the McManis protocol) and participants receive either placebo or bumetanide as a single dose on two different occasions. Electrophysiological measurements are used to assess the severity and the duration of the attack.

Results: The results of the first six participants will be presented. There were no serious adverse events. Vital signs and serum potassium levels were stable over a period of 4 hours after treatment intake in all participants. Outcome measurements will be discussed.

Conclusions: This is the first time bumetanide is utilised as a treatment option for patients with hypoPP. Interim results indicate that bumetanide is safe to use as a single dose in this patient group. The McManis test, used here as a procedure and an outcome measurement in a clinical trial for the first time, is well tolerated and produces reliable results. 